\title{
Nivolumab-Induced Granuloma Annulare
}

\author{
Bilal Fawaz, MD; Analisa Halpern, MD
}

\section{PRACTICE POINTS}

- Immune-related adverse events (irAEs) frequently occur in patients on immunotherapy, with the skin representing the most common site of involvement.

- Although rare, granulomatous reactions such as granuloma annulare increasingly are recognized as potential irAEs.

- Clinicians should be aware of this novel association to accurately diagnose and effectively treat adverse reactions in patients receiving immunotherapy.

Immune-checkpoint inhibitors (ICls) such as pembrolizumab and nivolumab have transformed oncologic therapeutic modalities. By disrupting tumor-induced immunosuppression, ICls have facilitated effective treatment of numerous malignancies, including metastatic melanoma; however, due to the ensuing disturbance of the immune system, various immune-mediated adverse reactions have been reported with $\mathrm{ICl}$ therapy. We present a case of nivolumab-induced granuloma annulare in a 54-year-old woman. Recognition of the association between $\mathrm{ICI}$ therapy and cutaneous adverse reactions is crucial to ensure accurate diagnosis and adequate treatment of such reactions in patients taking $\mathrm{ICls}$.

Cutis. 2021;107:E2-E4.

$\longrightarrow$ ranuloma annulare (GA) is a benign, cutaneous, granulomatous disease of unclear etiology. Typically, GA presents in young adults as asymptomatic, annular, flesh-colored to pink papules and plaques, commonly on the upper and lower extremities. Histologically, GA is characterized by mucin deposition, palisading or an interstitial granulomatous pattern, and collagen and elastic fiber degeneration. ${ }^{1}$

Granuloma annulare has been associated with various medications and medical conditions, including diabetes mellitus, hyperlipidemia, thyroid disease, and HIV. ${ }^{1}$ More recently, immune-checkpoint inhibitors (ICIs) have been reported to trigger GA. ${ }^{2}$ We report a case of nivolumab-induced GA in a 54-year-old woman.

\section{Case Report}

A 54-year-old woman presented with an itchy rash on the upper extremities, face, and chest of 4 months' duration. The patient noted that the rash started on the hands and progressed to include the arms, face, and chest. She also reported associated mild tenderness. She had a history of stage IV non-small-cell lung carcinoma with metastases to the ribs and adrenal glands. She had been started on biweekly intravenous infusions of the ICI nivolumab by her oncologist approximately 1 year prior to the current presentation after failing a course of conventional chemotherapy. The most recent positron emission tomography-computed tomography scan 1 month prior to presentation showed a stable lung mass with radiologic disappearance of metastases, indicating a favorable response to nivolumab. The patient also had a history of hypothyroidism and depression, which were treated with oral levothyroxine $75 \mu \mathrm{g}$ once daily and oral sertraline $50 \mathrm{mg}$ once daily, respectively, both for longer than 5 years.

Physical examination revealed annular, erythematous, flat-topped papules, some with surmounting fine scale, coalescing into larger plaques along the dorsal surface of the hands and arms (Figure 1) as well as the forehead and chest. A biopsy of a papule on the dorsal aspect of

From the Division of Dermatology, Cooper Medical School, Rowan University, Camden, New Jersey.

The authors report no conflict of interest.

Correspondence: Bilal Fawaz, MD, Division of Dermatology, Cooper Medical School of Rowan University, 3 Cooper Plaza, Ste 504, Camden, NJ 08103 (bfawaz7@gmail.com).

doi: 10.12788 /cutis. 0281 
the left hand revealed nodules of histiocytes admixed with Langerhans giant cells within the dermis; mucin was noted centrally within some nodules (Figure 2). Periodic acid-Schiff staining was negative for fungal elements compared to control. Polarization of the specimen was negative for foreign bodies. The biopsy findings therefore were consistent with a diagnosis of GA.

A 3-month treatment course of betamethasone dipropionate $0.05 \%$ cream twice daily failed. Narrowband UVB phototherapy was then initiated at 3 sessions weekly. The eruption of GA improved after 3 months of phototherapy. Subsequently, the patient was lost to follow-up.

\section{Comment}

Discovery of specific immune checkpoints in tumorinduced immunosuppression revolutionized oncologic therapy. An example is the programmed cell-death protein 1 (PD-1) receptor that is expressed on activated immune cells, including T cells and macrophages. ${ }^{3,4}$ Upon binding to the PD-1 ligand (PD-L1), T-cell proliferation
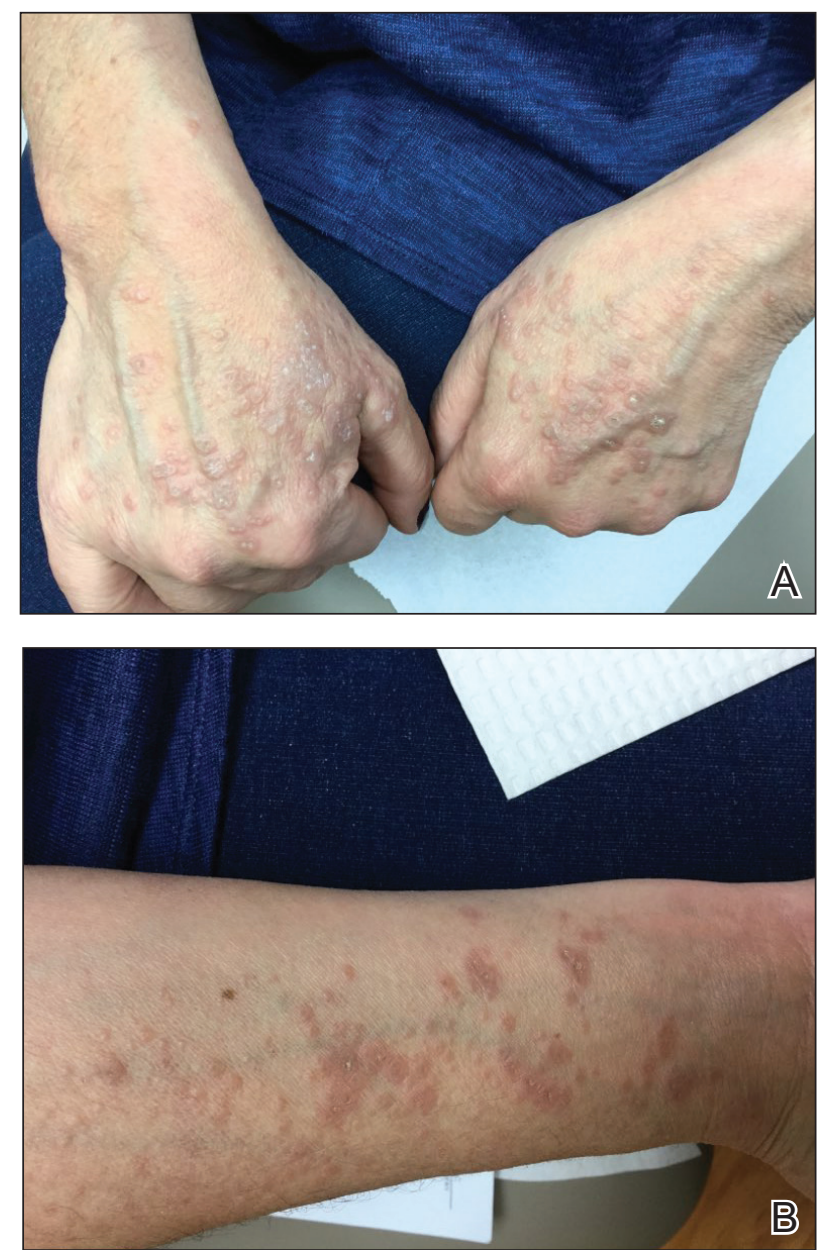

FIGURE 1. A and B, Annular, erythematous, flat-topped papules, some with surmounting fine scale, coalescing into larger plaques on the dorsal aspects of the hands and arms, respectively. is inhibited, resulting in downregulation of the immune response. As a result, tumor cells have evolved to overexpress PD-L1 to evade immunologic detection. ${ }^{3}$ Nivolumab, a fully human IgG4 antibody to PD-1, has emerged along with other ICIs as effective treatments for numerous cancers, including melanoma and non-small-cell lung cancer. By disrupting downregulation of $\mathrm{T}$ cells, ICIs improve immune-mediated antitumor activity. ${ }^{3}$

However, the resulting immunologic disturbance by ICIs has been reported to induce various cutaneous and systemic immune-mediated adverse reactions, including granulomatous reactions such as sarcoidosis, GA, and a cutaneous sarcoidlike granulomatous reaction. 1,2,5,6 Our patient represents a rare case of nivolumab-induced GA.

Recent evidence suggests that GA might be caused in part by a cell-mediated hypersensitivity reaction that is regulated by a helper $\mathrm{T}$ cell subset 1 inflammatory reaction. Through release of cytokines by activated $\mathrm{CD}^{+} \mathrm{T}$ cells, macrophages are recruited, forming the granulomatous pattern and secreting enzymes that can degrade connective tissue. Nivolumab and other ICIs can thus trigger this reaction because their blockade of PD-1 enhances T cellmediated immune reactions. ${ }^{2}$ In addition, because macrophages themselves also express PD-1, ICIs can directly enhance macrophage recruitment and proliferation, further increasing the risk of a granulomatous reaction. ${ }^{4}$

Interestingly, cutaneous adverse reactions to nivolumab have been associated with improved survival in melanoma patients. ${ }^{7}$ The nature of this association with granulomatous reactions in general and with GA specifically remains to be determined.

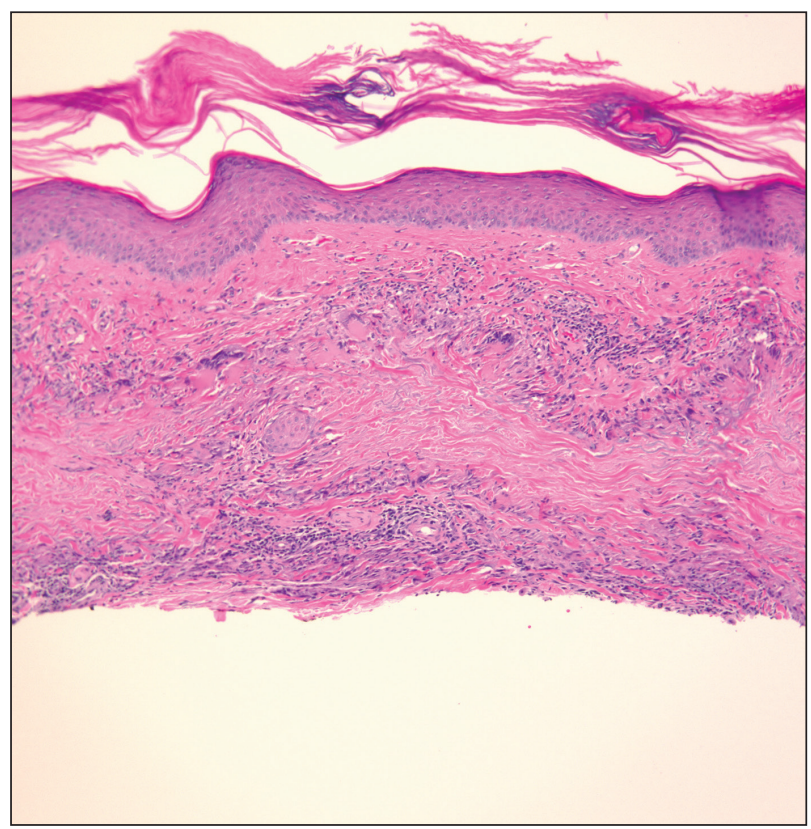

FIGURE 2. Within the dermis, histiocytic nodules were admixed with Langerhans giant cells with central mucin (H\&E, original magnification $\times 10$ ). 


\section{Conclusion}

Since the approval of the first PD-1 inhibitors, pembrolizumab and nivolumab, in 2014, other ICIs targeting the immune checkpoint pathway have been developed. Newer agents targeting PD-L1 (avelumab, atezolizumab, and durvalumab) were recently approved. Additionally, cemiplimab, another PD-1 inhibitor, was approved by the US Food and Drug Administration in 2018 for the treatment of advanced cutaneous squamous cell carcinoma. ${ }^{8}$ Indications for all ICIs also have expanded considerably. ${ }^{3}$ Therefore, the incidence of immune-mediated adverse reactions, including GA, is bound to increase. Physicians should be cognizant of this association to accurately diagnose and effectively treat adverse reactions in patients who are taking ICIs.

\section{REFERENCES}

1. Piette EW, Rosenbach M. Granuloma annulare: pathogenesis, disease associations and triggers, and therapeutic options. J Am Acad Dermatol. 2016;75:467-479. doi:10.1016/j.jaad.2015.03.055
2. Wu J, Kwong BY, Martires KJ, et al. Granuloma annulare associated with immune checkpoint inhibitors. J Eur Acad Dermatol. 2018;32:E124-E126. doi:10.1111/jdv.14617

3. Gong J, Chehrazi-Raffle A, Reddi S, et al. Development of PD-1 and PD-L1 inhibitors as a form of cancer immunotherapy: a comprehensive review of registration trials and future considerations. J Immunother Cancer. 2018;6:8. doi:10.1186/s40425-018-0316-z

4. Gordon SR, Maute RL, Dulken BW, et al. PD-1 expression by tumourassociated macrophages inhibits phagocytosis and tumour immunity. Nature. 2017;545:495-499. doi:10.1038/nature22396

5. Birnbaum MR, Ma MW, Fleisig S, et al. Nivolumab-related cutaneous sarcoidosis in a patient with lung adenocarcinoma. JAAD Case Rep. 2017;3:208-211. doi:10.1016/j.jdcr.2017.02.015

6. Danlos F-X, Pagès C, Baroudjian B, et al. Nivolumab-induced sarcoidlike granulomatous reaction in a patient with advanced melanoma. Chest. 2016;149:E133-E136. doi:10.1016/j.chest.2015.10.082

7. Freeman-Keller M, Kim Y, Cronin H, et al. Nivolumab in resected and unresectable metastatic melanoma: characteristics of immunerelated adverse events and association with outcomes. Clin Cancer Res. 2016;22:886-894. doi:10.1158/1078-0432.CCR-15-1136

8. Migden MR, Rischin D, Schmults CD, et al. PD-1 blockade with cemiplimab in advanced cutaneous squamous-cell carcinoma. N Engl J Med. 2018;379;341-351. doi:10.1056/NEJMoa1805131 